\title{
Diclofenac Sodium Stability in Simulated Gastrointestinal Fluids and the Use of MCM-41 Silica Carrier with Surface Modification to Achieve Innovative Delayed Release
}

\author{
Monica Chang Chuong ${ }^{1, *}$, Pornpawit Suriyamongkol', Ria Vashishth ${ }^{1}$, Ayushi Saxena', Charles Kelley ${ }^{2}$ \\ 'School of Pharmacy, MCPHS University, Boston, Massachusetts, US. \\ ${ }^{2}$ School of Arts and Sciences, MCPHS University, Boston, Massachusetts, US.
}

\begin{abstract}
Objectives: Porous silicon materials were first reported to be biocompatible in 1995. This project employed the evaluation of whether diclofenac sodium is acid labile or acid insoluble. Second, literature only reported $15 \%$ of alendronate entrapment in unmodified Mobil Composition of Matter No. 41 (MCM-41). Will functionalization of carry surface enhance drug entrapment? This project also assessed storage stability. Methods: Diclofenac sodium was subject to $0.1 \mathrm{~N}$ hydrochloric acid for $2 \mathrm{~h}$, then in phosphate buffer $\mathrm{pH} 6.8$ to determine the acid lability versus poor aqueous solubility. Entrapment parameters for unmodified MCM-41 were determined among stock concentration, driving medium, equilibration method, rate and duration. MCM-41 was then surface functionalized by tetraethylenepentamine/2-amino-2-methyl-1-propanol/ anhydrous alcohol and air dried into powder. Functionalized batch followed the same entrapment protocol as the unmodified MCM-41. Liquid chromatography and Fourier Transform Infrared were done at time zero and 3 months later. Results: Under the described conditions, the entrapment efficiency of diclofenac loaded amino-functionalized MCM-41 prepared by the immersion method was significant $(p<0.05)$ at $44.76 \% \pm 3.88 \%, n=3$.
\end{abstract}

The FT-IR spectra showed the loss of primary and protonated amino groups vibration in the 3-month-old unloaded-amino-modified MCM-41 group after being stored in room temperature. However, in the diclofenac loaded aminomodified-MCM-41 group, most amino group vibrations were still present. Conclusion: Diclofenac sodium is poorly aqueous soluble in simulating gastric fluid. Although diclofenac sodium is a cyclooxygenase-2 inhibitor, for the prolonged use in pain treatment, amino-surface-functionalization of MCM-41 may be performed to enhance drug entrapment and delay drug release due to electrostatic attraction and repulsion.

Key words: Delayed-release, Fourier Transform Infrared, high performance liquid chromatography, Mobil Composition of Matter No. 41 (MCM-41), mesoporous silica nanoparticles.

\section{Correspondence}

Dr. Monica C Chuong

Department of Pharmaceutical Sciences, MCPHS University, Boston, MA, USA

Phone No.: +1-617-735-1104

Email: monica.chuong@mcphs.edu

DOI: 10.5330/ijpi.2020.3.62

\section{INTRODUCTION}

Diclofenac, a non-steroidal anti-inflammatory drug commonly known for the indication of universal pain treatment, especially arthritis and menstrual pain, is primarily available as the sodium salt (Figure 1a) due to the low water solubility of diclofenac that ultimately affects the absorption and bioavailability. The notorious adverse effects include gastric irritation or bleeding. Literature suggested that diclofenac is somewhat acid labile/degraded in acidic condition..$^{1-3}$ Diclofenac sodium is formulated into delayed-release and extended-release oral tablets, but also as tropical gel, tropical solution, ophthalmic solution. ${ }^{4}$ The delayedrelease (also known as gastro-resistant) tablets which delay the drug release until reaching small intestine would be a solution to minimize its degradation in the stomach. Whether diclofenac sodium may be degraded in acid needs to be answered in pre-formulation step. However, another question to delve into is that why diclofenac sodium extendedrelease tablets was launched not long after the regulatory approval of its delayed-release tablets. ${ }^{4}$

A compound producing mesoporous silica was patented around 1970. The distinct properties of mesoporous silica nanoparticles include controlled porosity, morphology, particle size and high chemical stability, which make them highly attractive as ER drug carriers. Since MSNs were reported to be biocompatible in 1995, Mobil Composition of Matters
No. 41 (MCM-41), MCM-48 and Santa Barbara Amorphous 15 become commonly studied drug carriers due to the pore size ranging from 2 to $11 \mathrm{~nm}$ and their 2D-hexagonal and 3D-cubic structural characteristics. ${ }^{5}$ MCM-41 (Figure 1b) is synthesized by reacting tetraethyl orthosilicate with a template made of micellar rods, which resulted in a collection of nano-sized spheres or rods that are filled with a regular arrangement of pores. The template can then be removed by washing with a solvent adjusted to the proper $\mathrm{pH}$. Mesoporous particles can also be synthesized using other processes. However, (3-mercaptopropyl)trimethoxysilane is a better precursor than tetraethyl orthosilicate, which ensures more<smiles>O=C([O-])Cc1ccccc1Nc1c(Cl)cccc1Cl</smiles><smiles>C[Si](C)(O)O[Si](C)(C)O[Si](C)(O)O[Si](C)(C)O</smiles>

Figure 1: Chemical structures of (a) diclofenac sodium [6] and (b) MCM-41. 
uniform spheres and drastically reduces aggregation. Therefore, authors delved deeper into knowing more about the drug and the carrier. The first research aim of this project to answer was whether diclofenac sodium is acid labile like some literature claimed. ${ }^{1-3}$ Simulated gastric fluid was used to provide a rationale for selecting between delayedrelease and extended-release oral dosage forms in the preformulation stage. Second, entrapment efficiency of diclofenac sodium as the model drug in the MCM-41 carrier was assessed by varying parameters. The effect of functionalization of the pore surface of this carrier on the drug load and the physicochemical properties of the model drug were all within the scope of investigation.

\section{MATERIALS AND METHODS}

\section{Materials}

MCM-41 (MilliporeSigma, Lot MKCD5902 and Lot MKCD4544 twice) was purchased from MilliporeSigma (St. Louis, MO). Two separate orders of diclofenac sodium (both had the same lot number, R258D036, Alfa Aesar), acetonitrile (OmniSolv ${ }^{\circledast}$, Lot AXO142-1), Methanol (HPLC

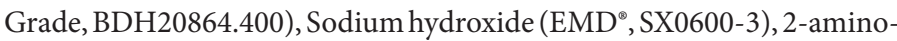
2-methyl-1-propanol (abbreviated as AMP, Merck KGaA, Lot S7133465 726), tetraethylenepentamine (abbreviated as TEPA, TCI America, Lot PXDZN JH) were obtained from VWR. Alcohol USP (PCCA, Lot C182544), potassium phosphate, monobasic NF (PCCA, Lot C121485), $85+\%$ phosphoric acid solution in water (ACROS ORGANICS, Lot B0531126) were purchased from PCCA. Ethyl alcohol 200 proof, (Pharmco, Lot C18E23002) was ordered from Fisher Scientific.

\section{Acid Lability versus Solubility Determination}

Three sample groups were prepared. Diclofenac sodium of $40 \mathrm{mg}$ was added to $300 \mathrm{~mL}$ of $0.1 \mathrm{~N}$ hydrochloric acid (as in simulated gastric fluid, Group A) and stood on bench top for $2 \mathrm{hr}$ with occasional stirs. ${ }^{6}$ After taking $3 \mathrm{~mL}$ to filter through a 0.22 micron syringe filter, 1-milliliter filtrate was injected into liquid chromatography analysis. The remaining Group A was mixed with tribasic sodium phosphate buffer in $3: 1 \mathrm{v} / \mathrm{v}$ ratio and adjusted $\mathrm{pH}$ to 6.8 with $2.0 \mathrm{~N} \mathrm{NaOH}$ solution (as in the simulated enteric fluid; Group B). Group C was prepared by adding 40 $\mathrm{mg}$ of diclofenac sodium directly into $400 \mathrm{~mL}$ sodium phosphate buffer, $\mathrm{pH} 6.8$ to serve as the control group. Visual inspection was done to assess any undissolved drug fine particles. LC analysis were performed using Group B and Group C samples.

\section{HPLC Method}

Agilent Series 1100 (Hewlett Packard) used in the project contained Vacuum Degasser, Binary Pump, Auto Sampler, Column Thermostated Compartment and Variable Wavelength Detector. The HPLC assay was adopted from the Diclofenac Sodium ER Tablets Monograph in the United States Pharmacopeia, ${ }^{6}$ except we used Hypersil ODS $\mathrm{C}_{18}, 4.9$ $\mathrm{mm} \times 15 \mathrm{~cm}, 5 \mu \mathrm{m}$ column recommended by Bhattacharya SS et al. ${ }^{7}$ to obtain a more satisfactory chromatographic tailing. The mobile phase was the mixture of $0.025 \mathrm{M}$ monobasic potassium phosphate buffer and acetonitrile $(40: 60 \mathrm{v} / \mathrm{v})$ and $\mathrm{pH}$ adjusted to 3.0 with $85 \%$ phosphoric acid. The other LC conditions included injection volume of $10 \mu \mathrm{L}$, mobile phase flow rate of $1 \mathrm{~mL} / \mathrm{min}$ and detector wavelength of $276 \mathrm{~nm}$. System suitability was performed. The linearity of diclofenac sodium in water was found in the range between 0.09 to $0.9 \mathrm{mg} / \mathrm{mL}\left(R^{2}=0.9998\right.$, $n=3)$. For diclofenac sodium in Alcohol USP, the linearity of standard calibration curve was found ranged from 0.04 to $9.00 \mathrm{mg} / \mathrm{mL}\left(R^{2}=1.0\right.$, $n=3)$. However, the Gaussian peak shape was lost in the Alcohol group after $5 \mathrm{mg} / \mathrm{mL}$.

\section{Particle Sizing}

The particle as well as pore sizes of MCM 41 may be found in the literature. They are $300-500 \mathrm{~nm}$ and 2 to $10 \mathrm{~nm}$ respectively. ${ }^{5,8}$ However, the particle size of the model drug, diclofenac sodium, was unknown. Therefore, the particle size was measured using a 90Plus Nanoparticle Size Analyzer (Brookhaven Instruments, Holtsville, NY). Literature indicated diclofenac sodium is slightly soluble in acetonitrile, hydrochloric acid $\mathrm{pH} 1.1$ (0.1 N hydrochloric acid), as well as cyclohexane. ${ }^{9}$ Based on literature..$^{10}$ the drug has the least solubility $(<1 \mathrm{mg} / \mathrm{mL})$ should be used. Therefore, diclofenac sodium was dispersed in acetonitrile with diclofenac refractive index set at 1.66210 ; acetonitrile refractive index at 1.344 at $20^{\circ} \mathrm{C},{ }^{11}$ and the viscosity at 0.369 at $25^{\circ} \mathrm{C}^{12}$ to assess the particle size of diclofenac.

\section{Entrapment Efficiency Study}

Diclofenac entrapment in MCM-41 was studied in two major aspects; (1) investigation of the drug left available in the supernatant using liquid chromatography which was then used to calculate entrapment efficiency, and (2) analysis of Fourier Transform Infrared spectra of the drug entrapped MCM-41 samples versus the control spectra of MCM41 and diclofenac alone respectively. Experiments are briefly described as follows.

\section{MCM-41 Silica as Carrier to Entrap Diclofenac Using} Water vs. Alcohol USP as the Driving Medium

(a) Water as the Entrapment Driving Medium

Diclofenac sodium was dissolved in water to make into $9 \mathrm{mg} / \mathrm{mL}$ stock solution. Then $5 \mathrm{~mL}$ of this solution (containing $45 \mathrm{mg}$ of diclofenac sodium) was pipetted into respective containers containing MCM-41 to make into drug/carrier ratios as 1:1, 1:2, 1:3 and 1:4. Samples were next divided into subgroups and triplicate for each subgroup. They were for two equilibration methods and two speeds, shaking on a VWR Advanced Digital Shaker at $65 \mathrm{rpm}$ and $85 \mathrm{rpm}$ respectively for $24 \mathrm{~h}$; the last subgroup was magnetically stirred at the lowest speed (1 out of 10) of a THERMIX ${ }^{\circ}$ STIRRIR (model 120MR, Fisher Scientific) for 24 h. At the end of equilibration, each mixture was transferred to a $15-\mathrm{mL}$ test tube to centrifuge at $3,000 \mathrm{rpm}$ for $30 \mathrm{~min}$. The supernatant was diluted 10 fold with nanopure water before subjecting to HPLC analysis. (The concentrations of the drug in the supernatant indicated as the unentrapped drug concentration.) With the standard curve of diclofenac in water, the un-entrapped drug concentration was determined. The entrapment efficiency was calculated using Equation 1.

Entrapment efficiency $(\%)=\frac{\text { Total drug amount }- \text { Unentrapped }}{\text { Total drug amount }} \times 100 \% \quad$ Eq (1)

Alcohol USP serving as a driving medium was next assessed with the entrapment process being performed in the same manner as water being the driving medium.

\section{Effect of Diclofenac Stock Solution Concentration on Entrapment Efficiency}

Lower stock concentration $(3 \mathrm{mg} / \mathrm{mL})$ was prepared using the same method as preparing $9 \mathrm{mg} / \mathrm{mL}$ stock solution in terms of equilibrating methods, such as shaking versus magnetic stirring, speed, duration and drug-carrier ratios (1:2, 1:3 and 1:4).

\section{Methods for Fourier Transform Infrared to Determine Whether Diclofenac Being Entrapped in MCM-41 Silica}

After the samples being centrifuged for $30 \mathrm{~min}$, a small volume of supernatant $(1 \mathrm{~mL})$ was collected from each sample and subjected 
to HPLC for entrapment efficiency determination. The remaining supernatant and condensate of a centrifuged sample was re-mixed thoroughly and passed through vacuum-assisted filtration with a 0.22 $\mu \mathrm{m}$ membrane. It was rinsed again with $10 \mathrm{~mL}$ nanopure water and vacuumed. The damp powder was transferred onto a weighing boat and covered with a tissue wiper to air dry overnight in controlled room temperature. This MCM-41 entrapped diclofenac dry powder were then characterized by Fourier Transform Infared spectrometer with OMNIC ${ }^{\mathrm{m}}$ Series Software (Thermo Scientific). The purpose was to compare the MCM-41 entrapped with diclofenac powder with two control groups (the MCM-41 alone and diclofenac sodium alone) in the wave number region between $4000 \mathrm{~cm}^{-1}$ and $400 \mathrm{~cm}^{-1}$.

\section{Preparative Method for Amino-Modified MCM-41 Pore Surface}

The pore surface of the purchased MCM-41 carriers was modified in our lab. Tetraethylenepentamine (TEPA) and 2-amino-2-methyl-1propanol (AMP), $9 \mathrm{~mL}$ each, were mixed. The final volume was adjusted with anhydrous ethyl alcohol to $30 \mathrm{~mL}$ in a beaker. The mixture then was subjected to sonication for $30 \mathrm{~min}$ in an ultrasound bath at room temperature to promote thorough dispersion of TEPA and AMP. ${ }^{13-15}$ After that, $1 \mathrm{~g}$ of MCM-41 was added to the mixture and sonicated for an extra $4 \mathrm{~h}$. During this 4 -h period, the water inside the ultrasound water bath was replenished with cold water hourly to avoid drug being degraded by the heat generated by sonication. Next, suspension beakers were placed inside an incubator at $85^{\circ} \mathrm{C} \pm 2^{\circ} \mathrm{C}$ for $20 \mathrm{~h}$ for the amine to bond the silanol groups on the pore surface of MCM-41. ${ }^{13-15}$ The beaker content was washed with $10 \mathrm{~mL}$ anhydrous ethanol and vacuum-filtered through $0.22 \mu \mathrm{m}$ filter membrane to collect the damp powder product. Immediately, the damp powder was transferred to another glass beaker to dry at $75^{\circ} \mathrm{C}$ overnight. Once the dried amine-modified MCM-41 was harvested, the powder was used for the entrapment efficiency analysis by loading diclofenac sodium and nanopure water the next day using the same equilibrium method as mentioned in Section 2.5.1.

\section{Three-Month Drug Entrapment of Conventional and Amino-Modified MCM-41}

The dry powder of diclofenac entrapped amino-modified MCM-41 was used for Fourier Transform Infrared spectrometer evaluation at timezero and three-month storage after being placed on weighing boats covered with wiper tissue at controlled room temperature. The untreated MCM-41 (from the vendor) and the amino-modified MCM-41 dried powder without drug load served as control groups individually.

\section{Statistics}

Excel 2016 worksheet (Microsoft) was used to manage raw data including the computation of the means and standard deviations of chromatographic AUCs. The $t$-test in Excel statistics was applied as two group comparison when normality and equal variance met. Population differences are considered significant at $p<0.05$.

\section{RESULTS}

\section{Acid Lability versus Aqueous Insolubility of Diclofenac Sodium}

A very small detected chromatographic peak was detected in Group A sample with retention time of $1.6 \mathrm{~min}$. which suggested that undissolved diclofenac in $0.1 \mathrm{~N}$ hydrochloride acid was filtered through a syringe filter. On the other hand, a significant peak of diclofenac at the retention time of 4 min appeared in Group B and Group C chromatograms. The AUC and concentrations of diclofenac were also similar between Groups
$\mathrm{B}$ and $\mathrm{C}(2183.5 \pm 45.5,0.097 \pm 0.002 \mathrm{mg} / \mathrm{mL}, 2218.4 \pm 26.6,0.098 \pm 0.001$ $\mathrm{mg} / \mathrm{mL}, n=3$ ). The data did not find diclofenac sodium degrade in $0.1 \mathrm{~N}$ hydrochloric acid, therefore diclofenac was proven not acid labile up to the experimental time, $2 \mathrm{hr}$, but it is poorly soluble in $0.1 \mathrm{~N} \mathrm{HCl}$.

\section{Particle Size Studies}

Using NanoBrook 90Plus Particle Size Analyzer, the effective diameter of diclofenac sodium particles in acetonitrile was found to be $789.5 \pm 242.5$ $\mathrm{nm}$ (3 readings on each sample run, $n=4)$.

\section{Entrapment Efficiency Study in Pre-Formulation Phase}

Diclofenac and MCM-41 entrapment study was performed in two ways. First, it was to investigate the drug in the supernatant using HPLC and the established standard curve to calculate entrapment efficiency. Second, it was to compare the FTIR spectra of the drug entrapped MCM-41 samples versus two controls (untreated MCM-41 and diclofenac sodium respectively).

\section{MCM-41 Silica as Carrier to Entrap Diclofenac using Water vs. Alcohol USP as the Driving Medium}

\section{In Water and Two Entrapment Methods (Stirring and Shaking)}

The entrapment efficiency of diclofenac in MCM-41 of 1:1, 1:2, 1:3 and 1:4 drug-to-carrier ratio samples using magnetic stirring for $24 \mathrm{~h}$ thus were not detected, $9.20 \%, 15.54 \%$ and $19.64 \%$ (Table $1 \mathrm{a}$ ). The entrapment efficiency using horizontal shaking for the same duration were $4.18 \%$, $12.32 \%, 21.17 \%(n=1)$ and $29.26 \% \pm 3.26 \%(n=4)$ (Table $1 \mathrm{~b})$. The data confirmed that horizontal shaking was more effective than magnetic stirring. However, the shaking rate (65 rpm vs $85 \mathrm{rpm}$ ) has no effect $(29.43 \% \pm 0.35 \%$ vs. $29.26 \% \pm 3.26 \%$ at $24 \mathrm{~h})$. The entrapment efficiency of 1:4 drug carrier using $3 \mathrm{mg} / \mathrm{mL}$ stock solution was not superior to that using $9 \mathrm{mg} / \mathrm{mL}$ stock solution at $24 \mathrm{~h}(19.44 \%$ vs $29.26 \%, p<0.05)$. Most diclofenac sodium was found present in Alcohol USP (99.08\%) without being entrapped in the carrier, MCM-41 (data not shown). Therefore, Alcohol USP was removed from the candidacy as a driving medium.

\section{FTIR Determination Whether Diclofenac being Entrapped in MCM-41}

The FTIR analysis results of the diclofenac sodium and unmodified MCM-41 as received from the vials sent by their vendors and samples of diclofenac sodium entrapped in the untreated MCM-41 (Figure 2). The stretching vibrations of Si-O-Si and Si-OH can be seen at $1065 \mathrm{~cm}^{-1}$ in MCM-41 (Figure 2b) and at 1066 and $966 \mathrm{~cm}^{-1}$ in diclofenac loaded untreated MCM-41 (Figure 2c) indicating the drug loading procedure did not affect the surface chemistry of the untreated MCM-41. ${ }^{16}$ The spectra of drug loaded MCM-41 (Figure 2c) shows a remarkable absence of the peaks which were observed in diclofenac suggesting that majority of diclofenac was entrapped in mesoporous silica nanoparticles as supported in literature. ${ }^{16,17}$ The peak at $792 \mathrm{~cm}^{-1}$ in diclofenac loaded unmodified MCM-41 spectra represents the fingerprint region of diclofenac (which is around 765 and $746 \mathrm{~cm}^{-1}$ in the spectra of pure diclofenac, Figure 2a) suggesting that very small amount of the drug adhered to the pore surface of untreated MCM- 41 .

\section{Amino-Modified MCM-41 Prepared by Immersion Method and HPLC Characterization}

Amine-modified MCM-41 of $180 \mathrm{mg}$ was added into to $5 \mathrm{~mL}$ of $9 \mathrm{mg} / \mathrm{mL}$ diclofenac sodium made of water as a driving medium. The resultant drug carrier ratio was 1:4. After being equilibrated on a horizontal shaker for $24 \mathrm{~h}$ and centrifuged 3,000 rpm for $30 \mathrm{~min}$, a sample 
of supernant after 10 -fold dilution was subjected to HPLC analysis. Under the same entrapment conditions larger drug AUC was present in a supernant chromatogram of an unmodified MCM-41 than that of an amine-modified group (suggesting as untrapped drug molecules). The entrapment percentage of diclofenac sodium in unmodified MCM41 was thus significantly lower than the amine-modified MCM-41 $(29.26 \% \pm 3.26 \%, n=4$ vs $44.76 \% \pm 3.88 \%, n=3, p<0.05)$. The controlled group was amine-modified MCM-41 in water without drug. The drug was eluted from the liquid chromatogram Hypersil ODS $\mathrm{C}_{18}$ column at the same time whether it was entrapped in the untreated or aminomodified MCM-41. Liquid chromatogram further proved that surface modification of MCM-41 as diclofenac carrier did not interfere with drug retention time (data not shown).

\section{FTIR Determination Whether Diclofenac Being Entrapped in or Surface Adhered on Amine-modified MCM-41}

The condensates of all three groups in amine-modified MCM-41 study prepared by immersion method were further air dried and used for FTIR. The remaining powders were stored at RT for stability study.

The FTIR spectra of diclofenac sodium pure compound, amine-modified MCM-41 without drug and amine-modified MCM-41 loaded with diclofenac sodium are shown in Figures $3 \mathrm{a}$ to $3 \mathrm{c}$. The amine-modified MCM-41 samples (Figure 3b) verified the presence of amino groups after modification. The asymmetric stretching vibrations $(\mathrm{Si}-\mathrm{O}-\mathrm{Si})$ appear at about $1054 \mathrm{~cm}^{-1}{ }^{16}$ The modification of MCM-41 samples with amine functional groups results in the vibration of primary amine groups $\left(-\mathrm{NH}_{2}\right)$ found at $1559 \mathrm{~cm}^{-1} .{ }^{16}$

\section{Stability Studies of Three-month-old Drug-Loaded in Amino-Modified MCM-41}

The 3-month-old batch of diclofenac-loaded in the untreated MCM-41 was noted color change from off-white to yellowish. Never the less, the

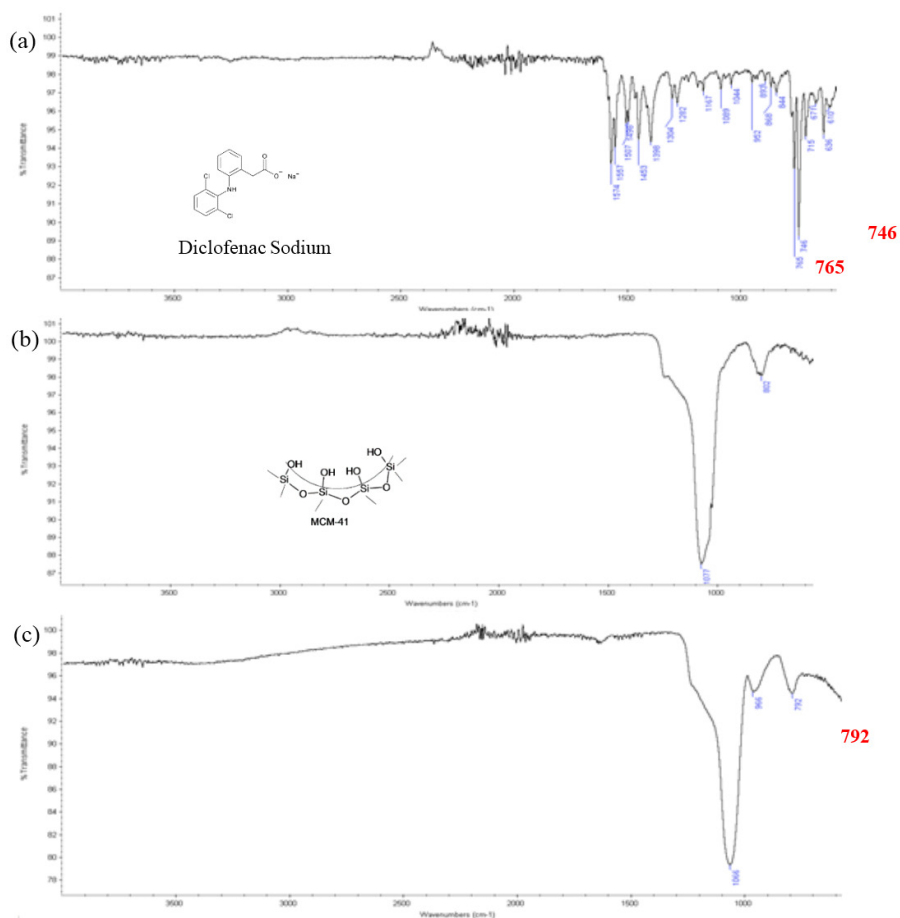

Figure 2: The FTIR scans of (a) diclofenac sodium, (b) untreated MCM-41 and (c) diclofenac sodium entrapped in the untreated MCM-41 (see text). powder of Diclofenac loaded amine-modified MCM-41 that had been stored under the same condition for 3 months remained as white.

The FT-IR spectra of amine modified MCM-41 post storage in a tightclose container under room temperature for three months (Figure 4b), when compared to the freshly prepared sample (Figure $4 \mathrm{a}$ ), showed the loss of primary and protonated amino group vibrations. However, the IR spectrum of 3-month-old diclofenac-loaded amino-modified MCM-41 stored on a weighing boat covered with tissue wiper in RT still displayed the primary and protonated amino group vibrations (Figure 5 bottom spectrum), but not in the group of amine modified MCM-41 without drug (Figure 5 top spectrum).

\section{DISCUSSION}

The solubility of diclofenac sodium in water was determined in the laboratory, because it has been reported differently in literatures including material safety data sheets. At the concentration of $10 \mathrm{mg} / \mathrm{mL}$, the solution became clear after sonication, but needle-like crystals were observed after being cooled to room temperature for some time. The same was not observed at $9 \mathrm{mg} / \mathrm{mL}$ sample. Thus, this project determined the solubility of diclofenac sodium in water at $9 \mathrm{mg} / \mathrm{mL}$.

The inner pore size of mesoporous silica nanoparticles used to entrap diclofenac sodium was in the mesopore size ranged from 2 to $10 \mathrm{~nm}^{6,8}$

(a)

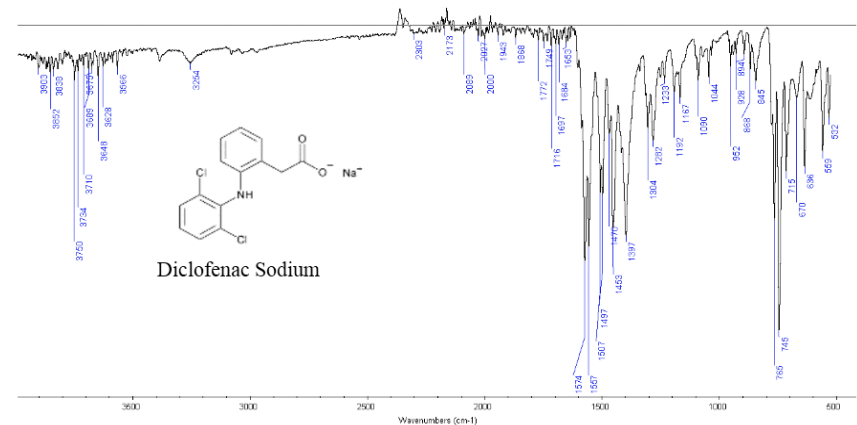

(b)

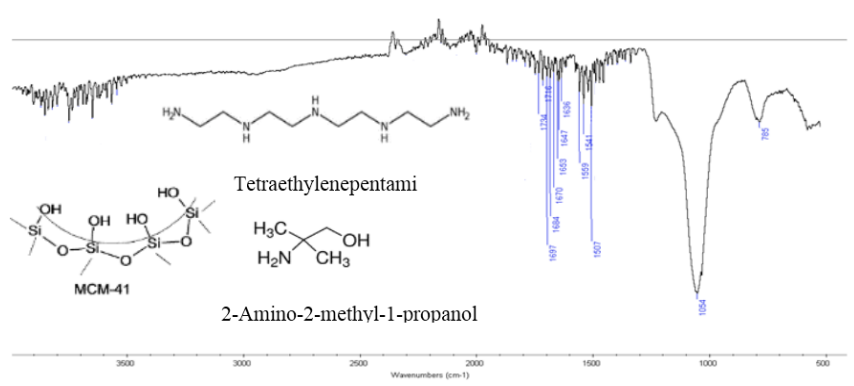

(c)

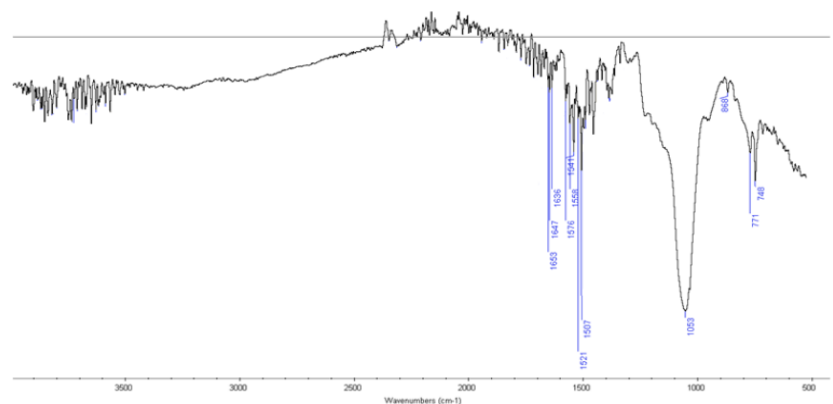

Figure 3: FTIR spectra of (a) diclofenac sodium pure compound, (b) aminemodified MCM-41 powder without drug load, and (c) diclofenac sodium entrapped in amine-modified MCM-41. 


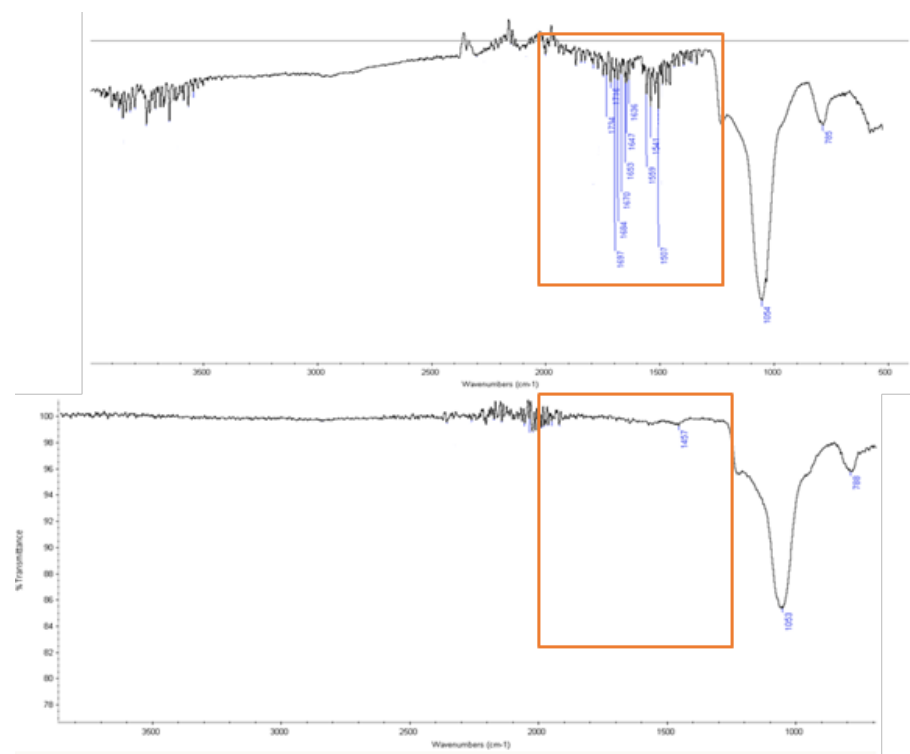

Figure 4: FT-IR spectra of fleshly prepared amino-modified MCM-41 (top) and a representative 3-month-old sample stored in a tight-close container at controlled room temperature (bottom). The primary and protonated amino group vibrations are squared ( $n=3$ each).

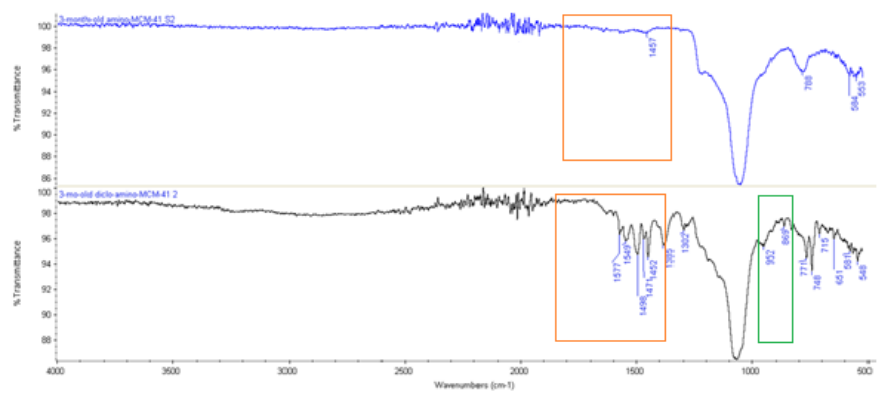

Figure 5: The FT-IR spectra of three-month stability study: amine modified MCM-41 without drug (top) and diclofenac loaded amino-MCM-41 (bottom). The fingerprint region of diclofenac is in the small green square and orange square the primary and protonated amino group vibrations are the large orange squares (bottom). Scans showed that without being loaded with the drug, the amino surface functionalized groups were lost (deleted) during storage (top spectrum).

was much smaller than the drug particles found in the particle size analysis, which was $789.5 \pm 242.5 \mathrm{~nm}(n=4)$. Thus, diclofenac sodium has to be completely dissolved in the driving medium (water) in order to enter the inner pore, entrap inside MCM-41 and form bond with its silanol groups.

The liquid chromatographic analysis supported the findings of Fourier Transform Infrared. For an unmodified MCM-41, during the drug entrapment process, two diclofenac molecules could form dimer to each other as seen in the ibuprofen molecule, ${ }^{17,18}$ which has similar structure as diclofenac sodium. However, its lower entrapment percentage suggests that the drug molecular affinity with silanol group of carrier is low. The entrapment percentage of diclofenac sodium in amino-modified MCM41 was significantly higher than that of diclofenac sodium in unmodified MCM- $41(44.76 \% \pm 3.88 \%$ vs $29.26 \% \pm 3.26 \%, n=3, p<0.05)$. This may be credited to the higher affinity to amine functional groups rather than the silanol group, which was also observed in ibuprofen molecule. ${ }^{17}$

For the patients who use diclofenac sodium for short durations diclofenac sodium extended-release tablets may be administered, since

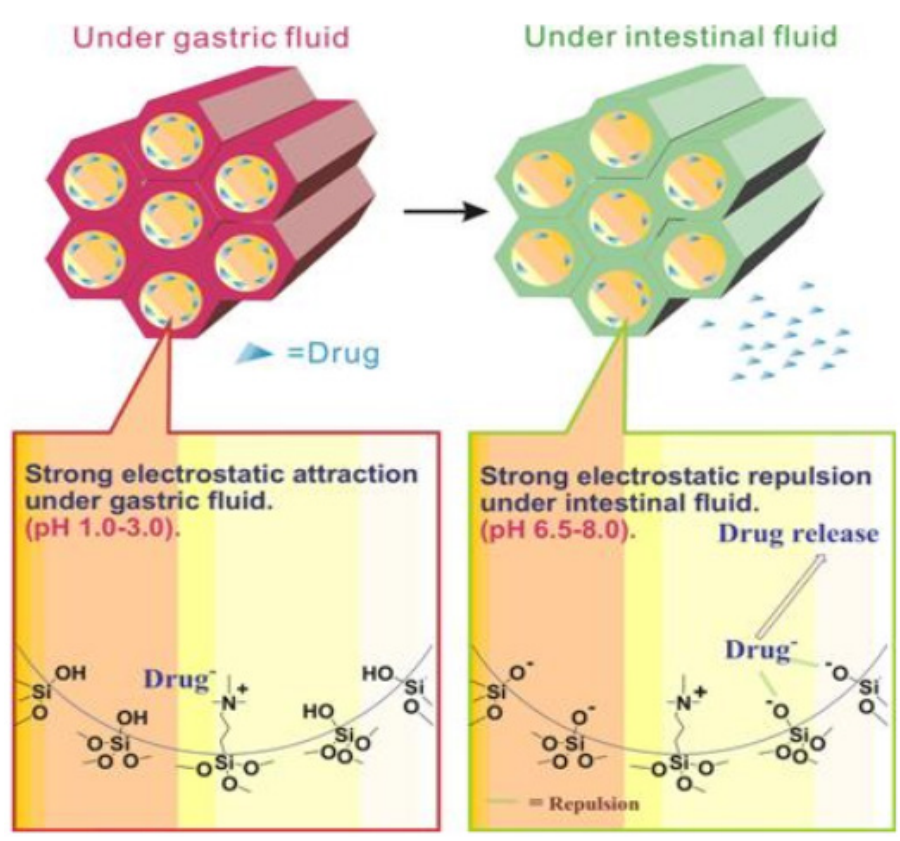

Figure 6: The interaction of an anionic drug with the amino-surface modified MCM-41 based on pH theory: under gastric fluid (left) and under intestinal fluid (right).

Table 1: The entrapment percentage of diclofenac in MCM-41 with water as the driving medium after 24 -hr equilibration by (a) magnetic stirrer and (b) horizontal motion shaker.

\begin{tabular}{cccc}
\hline (a) & & & \\
\hline $\begin{array}{c}\text { Drug-to- } \\
\text { carrier } \\
\text { Ratio }\end{array}$ & $\begin{array}{c}\text { Drug in } \\
\text { Supernatant } \\
(\mathrm{mg} / \mathrm{mL})\end{array}$ & $\begin{array}{c}\text { Drug in Supernatant } \\
\text { after correction }(\mathrm{mg} / \\
\mathrm{mL})^{*}\end{array}$ & $\begin{array}{c}\text { Entrapment } \\
(\%)\end{array}$ \\
\hline $1: 1$ & 0.91 & 9.05 & $0^{* *}$ \\
$1: 2$ & 0.82 & 8.17 & $9.20^{* *}$ \\
$1: 3$ & 0.76 & 7.60 & $15.54^{* *}$ \\
$1: 4$ & 0.72 & 7.23 & $19.64^{* *}$ \\
\hline
\end{tabular}

\begin{tabular}{cccc}
\hline (b) & & & \\
\hline $\begin{array}{c}\text { Drug-to- } \\
\text { carrier } \\
\text { Ratio }\end{array}$ & $\begin{array}{c}\text { Drug in } \\
\text { Supernatant } \\
(\mathrm{mg} / \mathrm{mL})\end{array}$ & $\begin{array}{c}\text { Drug in Supernatant } \\
\text { after correction }(\mathrm{mg} / \\
\mathrm{mL}) *\end{array}$ & $\begin{array}{c}\text { Entrapment } \\
(\%)\end{array}$ \\
\hline $1: 1$ & 0.86 & 8.62 & $4.18^{* *}$ \\
$1: 2$ & 0.79 & 7.89 & $12.32^{* *}$ \\
$1: 3$ & 0.71 & 7.09 & $21.17^{* *}$ \\
$1: 4$ & $0.64 \pm 0.03$ & $6.37 \pm 0.29$ & $29.26 \pm 3.26^{* * *}$ \\
\hline
\end{tabular}

* The concentration computed from the AUC of the drug peak was multiplied by the dilution factor of 10

${ }^{* *} n=1$

$* * * n=4$

it has less serious gastric adverse effects. ${ }^{18}$ However, for prolonged use, delayed release tablets are more suitable. This project supports a delayed release tablet formulation strategy by applying surface modification to carriers instead of using the conventional delayed release excipients, such as Eudragit ${ }^{\circledR}$, Acryl-EZE ${ }^{\circledR}$, Opdry Enteric and Sureteric ${ }^{\circledR}$ polymers as tablet coats. 
After oral administration, the presence of numerous hydrogen ions in the gastric fluid $(\mathrm{pH} 1.0$ - 3.0) results in the strong electrostatic attraction between the anionic/weak-acid drug (diclofenac) and the amine groups functionalized to the silanol groups of amino-modified MCM-41 (Figure 6, left). This rate-limiting step is expected to release less amount of diclofenac in the stomach to upset the organ. However, when moving to the intestines ( $\mathrm{pH} 6.5$ - 8.0), the silanol groups that were not functionalized by amino groups will get ionized to negative charge and create the strong electrostatic repulsion between themselves and the anionic molecules of diclofenac resulting in the drug release (Figure 6, right). This, yet, cannot be concluded until the further experiments are conducted.

\section{CONCLUSION}

The optimal entrapment parameters that contribute to the highest diclofenac loading efficiency in MCM-41 were the use of concentrated drug stock solution $(9 \mathrm{mg} / \mathrm{mL})$, water as the driving medium, 1:4 drug-carrier ratio and platform shaker as the equilibrating tool. The entrapment efficiency was found to be $29.26 \% \pm 3.26 \%$. Amine surface modification was further performed in order to modify the silanol groups in the mesopores of MCM-41 with amine groups and increase the affinity of diclofenac, weak acidic drug, surfaces of mesopores, ultimately improving the drug entrapment efficiency, $44.76 \% \pm 3.88 \%$ ( $p$ $<0.5$ ). The immersion method was proven a successful way to modify MCM-41 pore surface as seen in both FTIR spectra and the HPLC chromatograms. Finally yet importantly, drug entrapment should apply to amino-modified MCM-41 soon after the process. As seen in the FTIR spectra of the three-month stability study at controlled room temperatures, the surface modified amino groups may be deleted if drug is not soon loaded.

\section{REFERENCES}

1. Donnelly R, Pascuet E, Ma C, Vaillancourt R. Stability of Diclofenac Sodium Oral Suspensions Packaged in Amber Polyvinyl Chloride Bottles. The Canadian J Hospital Pharmacy. 2010;63(1):25-30

2. Wang $Y$, Huang $H$, Zhang $C$, Tang $Y$, Li J, Tang $X$, et al. Preparation of Highly
Stable Diclofenac Potassium Pellet with Microcrystalline Cellulose by Extrusion-Spheronization. Asian J Pharm Sci. 2013;8(6):356-61.

3. Wang F, Finnin J, Tait C, Quirk S, Chekhtman I, Donohue A, et al. The Hydrolysis of Diclofenac Esters: Synthetic Prodrug Building Blocks for Biodegradable Drug-Polymer Conjugates. J Pharm Sci. 2016;105(2):773-85.

4. U.S. Food and Drug Administration. Diclofenac sodium. Orange book: Approved Drug Products with Therapeutic Equivalence Evaluations. 2020. Available from https://www.accessdata.fda.gov/scripts/cder/ob/index.cfm

5. Hartmann M, Jung D. Immobilization of proteins and enzymes, mesoporous supports. Upstream Industrial Biotechnology: Equipment, Process Design, Sensing, Control and cGMP Operations, First Ed. New Jersey: John Wiley and Sons, Inc. 2013;2:1148.

6. The United States Pharmacopeial Convention Diclofenac Sodium Extended-Release Tablets, and Diclofenac Sodium and Misoprostol Delayed-Release Tablets Monographs, and General Chapters: <711> Dissolution. USP43-NF38. Maryland: Pharmacopeia. 2020;1348, 1350, 6945.

7. Bhattacharya S, Banerjee S, Ghosh A, Chattopadhyay P, Verma A, Ghosh A. A RP-HPLC Method for Quantification of Diclofenac Sodium Released from Biological Macromolecules. Int J Biol Macromol. 2013;58:354-9.

8. Ng E, Goh J, Ling TC, Mukti RR. Eco-friendly synthesis for MCM-41 nanoporous materials using the non-reacted reagents in mother liquor. Nanoscale Res Lett. 2013;8(1):120

9. O'Neil MJ. Diclofenac Sodium. The Merck Index: An Encyclopedia of Chemicals, Drugs, and Biologicals. Royal Society of Chemistry. $15^{\text {th }}$ ed. 2103.

10. Diclofenac. Royal Society of Chemistry. 2020

11. Acetonitrile: Sigma-Aldrich Acetonitrile. Millipore Sigma. 2018.

12. Barnes P, Simon J, Martin V. Peak Shape Functions: Gaussian. Birkbeck College: University of London. 2006.

13. Shodhganga Contributors. Dasatinib and Mesoporous MCM-41 MSNs. Shodhganga: A reservoir of Indian theses. 2020. shodhganga.inflibnet.ac.in/bitstream/10603/34727/12/12_dasatiniband\%20mcm.pdf

14. Lang Y, Finn D, Pandit A, Walsh P. Pharmacological Activity of Ibuprofen Released from Mesoporous Silica. J Materials Science: Materials in Medicine. 2011;23(1):73-80.

15. Szegedi A, Popova M, Goshev I, Klébert S, Mihály J. Controlled Drug Release on Amine Functionalized Spherical MCM-41. J Solid State Chemistry. 2012;194:257-63.

16. Silverstein RM, Webster FX, Kiemle DJ, Bryce DL. Infared Spectroscopy. Spectormetric Identification of Organic Compounds. New Jersey: Wiley. 2015;80,123.

17. Warner T, Giuliano F, Vojnovic I, Bukasa A, Mitchell J, Vane J. Nonsteroid Drug Selectivities for Cyclo-oxygenase-1 rather than cyclo-oxygenase-2 are associated with human gastrointestinal toxicity: A full in vitro analysis. Proceedings of the National Academy of Sciences. 1999;96(13):7563-8.

18. Knights K, Mangoni A, Miners J. Non-selective Nonsteroidal Anti-inflammatory Drugs and Cardiovascular Events: Is Aldosterone the Silent Partner in Crime? British J Clinical Pharmacol. 2006:61(6):738-40.

Article History: Submission Date : 07-06-2020; Revised Date : 22-07-2020; Acceptance Date : 15-08-2020.

Cite this article: Chuong MC, Suriyamongkol P, Vashishth R, Saxena A, Kelley C. Diclofenac Sodium Stability in Simulated Gastrointestinal Fluids and the Use of MCM-41 Silica Carrier with Surface Modification to Achieve Innovative Delayed Release. Int. J. Pharm. Investigation, 2020;10(3):351-6. 